\title{
多孔钼钛氧化物的自支撑制备及其结构转变
}

\author{
李力成 ${ }^{1}$, 何甜甜 ${ }^{1}$, 赵学娟 ${ }^{2}$, 钱 祺 ${ }^{1}$, 王 砳 ${ }^{1}$, 李小保 ${ }^{1}$
}

(1. 南京林业大学 化学工程学院, 南京 210037; 2. 南京工程学院 材料工程学院, 南京 211167)

摘 要: 通过 $\mathrm{MoO}_{3}$ 与 $\mathrm{TiO}_{2}$ 相互支撑的方法制备了一系列多孔钼钛氧化物, 并在此基础上研究了该材料结构在随焙 烧温度变化过程中的转变机制, 通过 XRD、BET、FESEM、TG/DTG 等表征分析, 当焙烧温度低于 $600^{\circ} \mathrm{C}$ 时, $\mathrm{MoO}_{3}$ 呈固体状态, 通过 $\mathrm{MoO}_{3}$ 与 $\mathrm{TiO}_{2}$ 相互支撑可以制备出比表面积高达 $182 \mathrm{~m}^{2} / \mathrm{g}$ 的介孔钼钛氧化物, 可负载更多分散 良好的 $\mathrm{MoO}_{3}$, 其加氢脱硫性能显著优于常规浸渍法制备的催化材料; 当焙烧温度高于 $600^{\circ} \mathrm{C}$ 时, $\mathrm{MoO}_{3}$ 呈熔融状态, “自支撑效应”消失，钼钛氧化物孔结构发生埋塌。

关 键 词: 钿钛氧化物; 自支撑; 比表面积; 结构转变; 加氢脱硫

中图分类号: TQ34 文献标识码: A

\section{Self Supported Synthesis of Porous Molybdenum-titanium Oxide and the Resulting Structural Transformation}

\author{
LI Li-Cheng ${ }^{1}$, HE Tian-Tian ${ }^{1}$, ZHAO Xue-Juan ${ }^{2}$, QIAN Qi ${ }^{1}$, WANG Lei ${ }^{1}$, LI Xiao-Bao ${ }^{1}$
}

(1. College of Chemical Engineering, Nanjing Forestry University, Nanjing 210037, China; 2. School of Materials Science and Engineering, Nanjing Institute of Technology, Nanjing 211167, China)

\begin{abstract}
A series of porous molybdenum-titanium oxide $\left(\mathrm{Mo}-\mathrm{TiO}_{2}\right)$ were prepared by mutual support of $\mathrm{MoO}_{3}$ and $\mathrm{TiO}_{2}$, and the resulting structural transformation with calcination temperature was also studied in the present study. The as-prepared samples were characterized mainly by XRD, BET, FESEM, and TG/DSC. When the calcination temperature was lower than $600^{\circ} \mathrm{C}, \mathrm{MoO}_{3}$ can maintain solid state. Mesoporous Mo-TiO ${ }_{2}$ with surface area of $182 \mathrm{~m}^{2} / \mathrm{g}$ was obtained via mutual support of $\mathrm{MoO}_{3}$ and $\mathrm{TiO}_{2}$. The oxide loaded by more well-dispersed $\mathrm{MoO}_{3}$ possessed significantly better hydrodesulfurization performance than that prepared by impregnation did. As $\mathrm{MoO}_{3}$ was fused at above $600^{\circ} \mathrm{C}$, "self supported effect” was disappeared and the porous structure of $\mathrm{Mo}^{-\mathrm{TiO}}{ }_{2}$ collapsed finally.
\end{abstract}

Key words: molybdenum-titanium oxide; self support; surface area; structural transformation; hydrodesulfurization

以 $\mathrm{TiO}_{2}$ 为载体的钼钛氧化物因其优良的催化 性能在加氢、选择性催化、光催化等反应体系中受 到广泛关注 ${ }^{[1-3]}$ 。然而, 常规 $\mathrm{TiO}_{2}$ 的比表面积比其 他传统载体材料(例如 $\mathrm{SiO}_{2}$ 和 $\mathrm{Al}_{2} \mathrm{O}_{3}$ )小, 限制了活 性组分的负载量, 使其难以为反应提供足够多的
活性位点。另外，由于 $\mathrm{TiO}_{2}$ 热稳定性低、孔结构易 在高温下坞塌 ${ }^{[4-5]}$, 因而通过高温焙烧难以得到大 比表面积多孔结构的 $\mathrm{TiO}_{2}$ 基催化材料。到目前为 止, 提高 $\mathrm{TiO}_{2}$ 的比表面积依然是 $\mathrm{TiO}_{2}$ 基催化材料 的研究热点。

收稿日期: 2016-01-28; 收到修改稿日期：2016-03-25

基金项目: 国家自然科学基金(21406118, 91434109, 91334202); 南京林业大学和南京工程学院人才启动基金(GXL2014036, YKJ201310); 江苏高校优势学科建设工程资助项目

National Natural Science Foundation of China (21406118, 91434109, 91334202); Startup Funding from Nanjing Forestry University (GXL2014036) and Nanjing Institute of Technology (YKJ201310); Priority Academic Program Development of Jiangsu Higher Education Institutions

作者简介: 李力成(1986-), 男, 博士, 讲师. E-mail: llc0024@yahoo.com 
在之前大量的研究工作中, 普遍采用溶胶一凝 胶法制得多孔 $\mathrm{TiO}_{2}$, 其比表面积可高达 $100 \mathrm{~m}^{2} / \mathrm{g}$ 以 上, 但该法使用的原料较为昂贵 ${ }^{[6-7]}$ 。此外, 模板法 也是较普遍采用的多孔材料制备方法, 该法先获得 具有高比表面积或者孔径均匀的非晶前驱体, 然 后移除模板得到晶态的多孔材料, 但是需要注意 模板去除过程对材料结构及其后续催化性能造成 的影响 ${ }^{[8-9]}$ 。由钛酸盐出发通过水热法可合成比表面 积达 $200 \mathrm{~m}^{2} / \mathrm{g}$ 以上的 $\mathrm{TiO}_{2}$ 纳米管, 但水热法条件苛

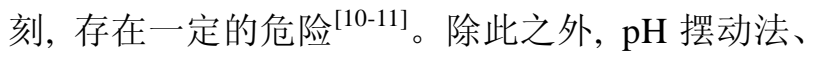
多步凝胶法等新方法也可在一定程度上改进 $\mathrm{TiO}_{2}$ 的结构 ${ }^{[12-13]}$ 。在我们前期研究中, 由 $\mathrm{TiO}_{2} \cdot n \mathrm{H}_{2} \mathrm{O}$ 和 $\mathrm{K}_{2} \mathrm{CO}_{3}$ 等原材料通过高温固相烧结制备了钛酸钾, 经离子交换获得多孔非晶态钛酸盐衍生物 $\left(\mathrm{H}_{2} \mathrm{Ti}_{2} \mathrm{O}_{5} \cdot x \mathrm{H}_{2} \mathrm{O}\right)$, 再进一步焙烧脱水后制得介孔 $\mathrm{TiO}_{2}$, 其比表面积可达 $100 \mathrm{~m}^{2} / \mathrm{g}$ 以上 ${ }^{[14]}$ 。然而, 高 比表面积 $\mathrm{TiO}_{2}$ 需要通过高温焙烧负载 $\mathrm{MoO}_{3}$, 由于 $\mathrm{TiO}_{2}$ 的热稳定性差, 多次高温焙烧会进一步减小 $\mathrm{TiO}_{2}$ 载体催化材料的比表面积。通过向 $\mathrm{TiO}_{2}$ 材料中 引入 $\mathrm{K} 、 \mathrm{Al} 、 \mathrm{Si}$ 等结构助剂可以有效提高其热稳定 性 ${ }^{[15-17]}$, 但是结构添加剂对催化活性中心的影响机 制尚未见文献报道。

相比之下，自支撑合成技术无需引入其他物 种、无需残留杂质影响, 可利用复合物自身各组分 相互支撑来保护材料的微观结构和提高材料的热稳 定性 ${ }^{[18-21]}$ 。因此, 本研究通过自支撑方法制备了介 孔钼钛氧化物, 探讨了氧化物结构与加氢脱硫性能 间的构效关系, 并进一步探讨了自支撑作用对钿钛 氧化物孔结构的影响。

\section{1 实验方法}

\section{1 钼钛氧化物的制备}

多孔钼钛氧化物 $\left(\mathrm{Mo}-\mathrm{TiO}_{2}\right)$ 是由一种多孔钛酸
盐衍生物 $\left(\mathrm{H}_{2} \mathrm{Ti}_{2} \mathrm{O}_{5} \cdot \mathrm{xH}_{2} \mathrm{O}\right)$ 制备而成，该材料制备过 程在前期工作中已有报道 ${ }^{[14]}$, 其结构数据如表 1 所示。

将干燥的钛酸盐衍生物加入到钼酸铵 $\left(\left(\mathrm{NH}_{4}\right)_{6} \mathrm{Mo}_{7} \mathrm{O}_{24} \cdot 4 \mathrm{H}_{2} \mathrm{O}\right)$ 水溶液中, 搅拌 $0.5 \mathrm{~h}$, 加热、 蒸干, 经不同温度焙烧之后得到多孔钼钛氧化物 $\left(\mathrm{Mo}-\mathrm{TiO}_{2}\right)$ 的前驱体, 记作 $\mathrm{Mo}(\mathrm{I})-\mathrm{TiO}_{2}(x), x$ 为焙烧温 度。然后, 将前驱体 $\mathrm{Mo}(\mathrm{I})-\mathrm{TiO}_{2}$ 置于氨水中, 溶解 前驱体中过量的 $\mathrm{MoO}_{3}$, 搅拌 $2 \mathrm{~h}$ 后过滤, 去离子水 洗涤沉淀物, 干燥后得到多孔 $\mathrm{Mo}-\mathrm{TiO}_{2}$, 标记为

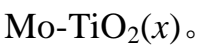

\section{2 表征}

采用 D8 Adavance 型 X 射线粉末衍射仪(XRD) 解析样品的晶相结构, 扫描范围 $5^{\circ} \sim 60^{\circ}$, 扫描步长 $0.05 \%$ step, 扫描速率 $0.2 \mathrm{~s} / \mathrm{step}$; 采用 TristarII $3020 \mathrm{M}$ 比表面孔隙吸附测定仪测定样品的比表面积、孔分 布和孔容; 热重分析(TGA-DTA, Model SDT 2960) 在 35 950 ${ }^{\circ} \mathrm{C}$ 的空气气氛中进行, 升温速率 $10 \mathrm{~mL} / \mathrm{min}$; 采用 Hitachi S-4800 场发射扫描电镜(FESEM)分析 样品的微观形貌, 操作电压 $5 \mathrm{kV}$ 。采用 ADVANT" $X P$ 型 $X$ 射线荧光光谱仪 $(X R F)$ 测定样品中 $\mathrm{MoO}_{3}$ 的含量。

\section{3 催化活性测试}

多孔钼钛氧化物的加氢脱硫性能测试在微型 固定床反应器上进行, 以二苯并噻吩 (DBT)作为脱 硫模型化合物, $1 \mathrm{wt} \% \mathrm{DBT} /$ 十氢萗作为模拟柴油反 应液(硫含量为 $1.770 \mathrm{mg} / \mathrm{g}$ ), 预硫化液为 $3 \% \mathrm{CS}_{2} /$ 十氢萗。先将催化剂预硫化 $10 \mathrm{~h}$, 温度为 $300^{\circ} \mathrm{C}$, 压 力为 $2 \mathrm{MPa}$, 氢油比 $(V / V)$ 为 600 , 体积空速为 $6 \mathrm{~h}^{-1}$ 。 待预硫化过程结束, 管路切换成反应液进行脱硫 反应。反应 $6 \mathrm{~h}$ 后开始采集样品, 每隔 $1 \mathrm{~h}$ 采集一 次。产物通过气相色谱检测, 采用归一法计算 DBT 的转化率。

表 1 不同钼钛氧化物的结构数据

Table 1 Structural data of various porous molybdenum-titanium oxides

\begin{tabular}{|c|c|c|c|c|c|c|}
\hline Samples & Crystalline phase & $\begin{array}{l}\text { Crystalline particle } \\
\text { size } / \mathrm{nm}^{\alpha}\end{array}$ & $\begin{array}{l}\text { Surface area } \\
/\left(\mathrm{m}^{2} \cdot \mathrm{g}^{-1}\right)\end{array}$ & $\begin{array}{l}\text { Pore volume } \\
/\left(\mathrm{cm}^{3} \cdot \mathrm{g}^{-1}\right)\end{array}$ & $\begin{array}{l}\text { Average pore } \\
\text { size / nm }\end{array}$ & $\begin{array}{c}\mathrm{MoO}_{3} \\
\text { content / \% }\end{array}$ \\
\hline Titanate derivate & Amorphous & I & 234 & 0.17 & 3.2 & l \\
\hline $\mathrm{TiO}_{2}$ & Anatase & $8.6^{\mathrm{A}}$ & 103 & 0.20 & 5.9 & l \\
\hline Mo- $\mathrm{TiO}_{2}(400)$ & Anatase & $4.7^{\mathrm{A}}$ & 182 & 0.14 & 3.7 & 16.1 \\
\hline Mo- $\mathrm{TiO}_{2}(500)$ & Anatase & $6.2^{\mathrm{A}}$ & 173 & 0.21 & 4.9 & 14.0 \\
\hline Mo- $\mathrm{TiO}_{2}(600)$ & Anatase/rutile & $14.1^{\mathrm{A}}, 19.3^{\mathrm{R}}$ & 39 & 0.16 & 14.1 & 9.2 \\
\hline Mo- $\mathrm{TiO}_{2}(700)$ & Rutile & $19.0^{\mathrm{R}}$ & 7.0 & 0.03 & l & 2.9 \\
\hline Mo- $\mathrm{TiO}_{2}(800)$ & Rutile & $21.5^{\mathrm{R}}$ & 3.2 & $<0.01$ & / & 2.5 \\
\hline
\end{tabular}

$\alpha$ : The crystalline particle size of anatase and rutile $\mathrm{TiO}_{2}$ of composites are calculated by Scherrer equation ${ }^{[22]}$. " $\mathrm{A}$ ” remarks the particle size of anatase $\mathrm{TiO}_{2}$ while " $\mathrm{R}$ " remarks the particle size of rutile $\mathrm{TiO}_{2}$ 


\section{2 结果与讨论}

\section{1 结构信息和催化性能}

所有样品的结构参数如表 1 所示, $\mathrm{Mo}-\mathrm{TiO}_{2}(400)$ 和 $\mathrm{Mo}-\mathrm{TiO}_{2}(500)$ 的比表面积分别为 $182 \mathrm{~m}^{2} / \mathrm{g}$ 和 $173 \mathrm{~m}^{2} / \mathrm{g}$, 两者的比表面积均显著高于没有自支撑 的 $\mathrm{TiO}_{2}\left(103 \mathrm{~m}^{2} / \mathrm{g}\right)$ 。图 1 显示 $\mathrm{Mo}-\mathrm{TiO}_{2}(400)$ 与 $\mathrm{Mo}^{-} \mathrm{TiO}_{2}$ (500)两者的 $\mathrm{N}_{2}$ 等温吸附脱附线基本重叠, 表明它们的孔结构相似; $\mathrm{Mo}^{-\mathrm{TiO}_{2}}(400)$ 和 Mo- $\mathrm{TiO}_{2}(500)$ 的 $\mathrm{N}_{2}$ 吸附主要发生在 $0.4<P / P_{0}<0.6$ 区 间，并且两者吸附脱附线中均存在明显的 $\mathrm{H} 1$ 型滞 后环, 这是典型的 IV 等温线特征, 表明两种氧化物 具有典型的介孔结构 ${ }^{[23]}$; 其他结构数据如孔容和平 均孔径等详见表 1 。当焙烧温度升至 $600^{\circ} \mathrm{C}$ 时, Mo- $\mathrm{TiO}_{2}(600)$ 的比表面积降到 $39 \mathrm{~m}^{2} / \mathrm{g}$, 明显低于 $\mathrm{Mo}^{-} \mathrm{TiO}_{2}(500)$; 随着焙烧温度进一步升高, 钼钛氧化
物的孔结构发生急剧埋塌, $\mathrm{Mo}-\mathrm{TiO}_{2}(700)$ 和 $\mathrm{Mo}-\mathrm{TiO}_{2}$ (800)的比表面积分别仅为 $7.0 \mathrm{~m}^{2} / \mathrm{g}$ 和 $3.2 \mathrm{~m}^{2} / \mathrm{g}$ 。

图 2 为多孔钼钛氧化物的 FESEM 照片。如图

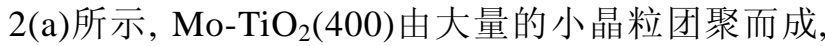
各晶粒间的空隙为 $\mathrm{Mo}-\mathrm{TiO}_{2}(400)$ 的孔道; $\mathrm{Mo}-\mathrm{TiO}_{2}$

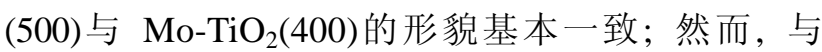

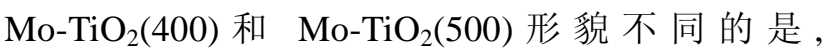
$\mathrm{Mo}^{-} \mathrm{TiO}_{2}(600)$ 的晶粒尺寸更大，且出现晶粒间部分 熔合的现象。当焙烧温度继续升高时, 在 $\mathrm{Mo}-\mathrm{TiO}_{2}$ (700)和 $\mathrm{Mo}^{-} \mathrm{TiO}_{2}(800)$ 中很难观察到分散状的晶粒, 晶粒相互间完全熔合。

由图 3 可知，当焙烧温度低于 $600^{\circ} \mathrm{C}$ ，自支撑合 成的氧化物 $\left(\mathrm{Mo}-\mathrm{TiO}_{2}(400), \mathrm{Mo}-\mathrm{TiO}_{2}(500)\right)$ 比表面积 高于没有支撑作用的 $\mathrm{TiO}_{2}$, 说明自支撑可以在高温 焙烧过程中有效地维持钼钛氧化物的孔结构。当焙 烧温度高于 $600{ }^{\circ} \mathrm{C}, \mathrm{Mo}-\mathrm{TiO}_{2}$ 和 $\mathrm{TiO}_{2}$ 的比表面积均有
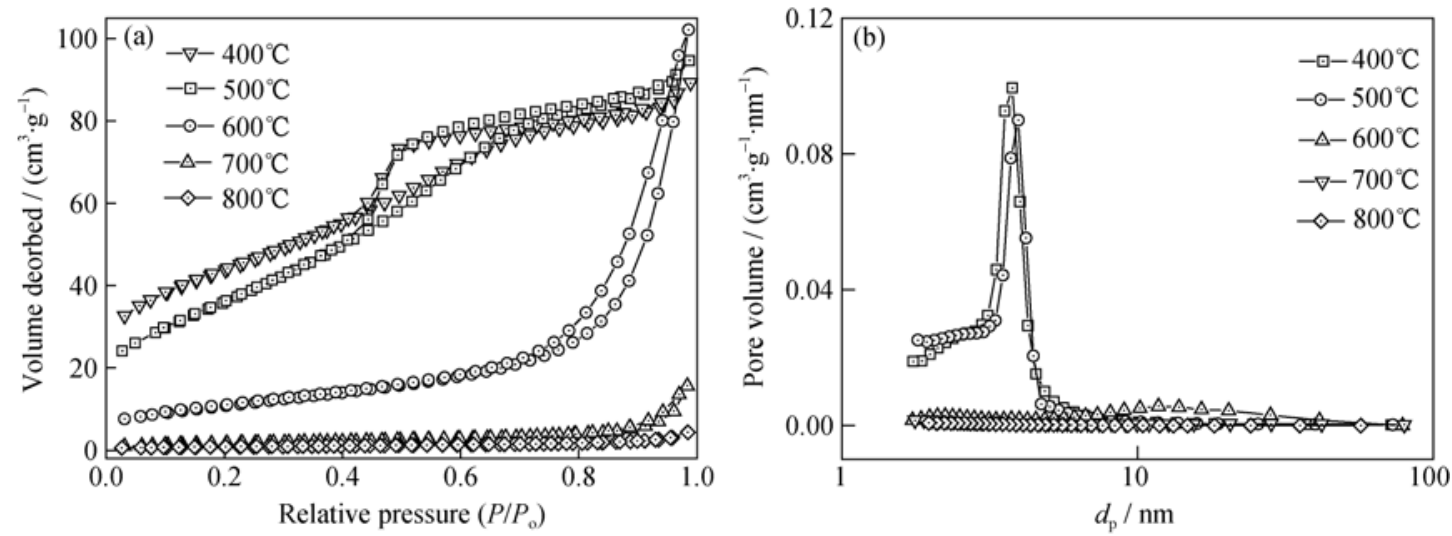

图 1 不同焙烧温度制备多孔钼钛氧化物的 $\mathrm{N}_{2}$ 吸附/脱附等温曲线(a)和孔径分布图(b)

Fig. $1 \mathrm{~N}_{2}$ adsorption-desorption isotherms (a) and pore size distributions (b) of porous $\mathrm{Mo}^{-\mathrm{TiO}_{2}}$ with different calcination temperatures
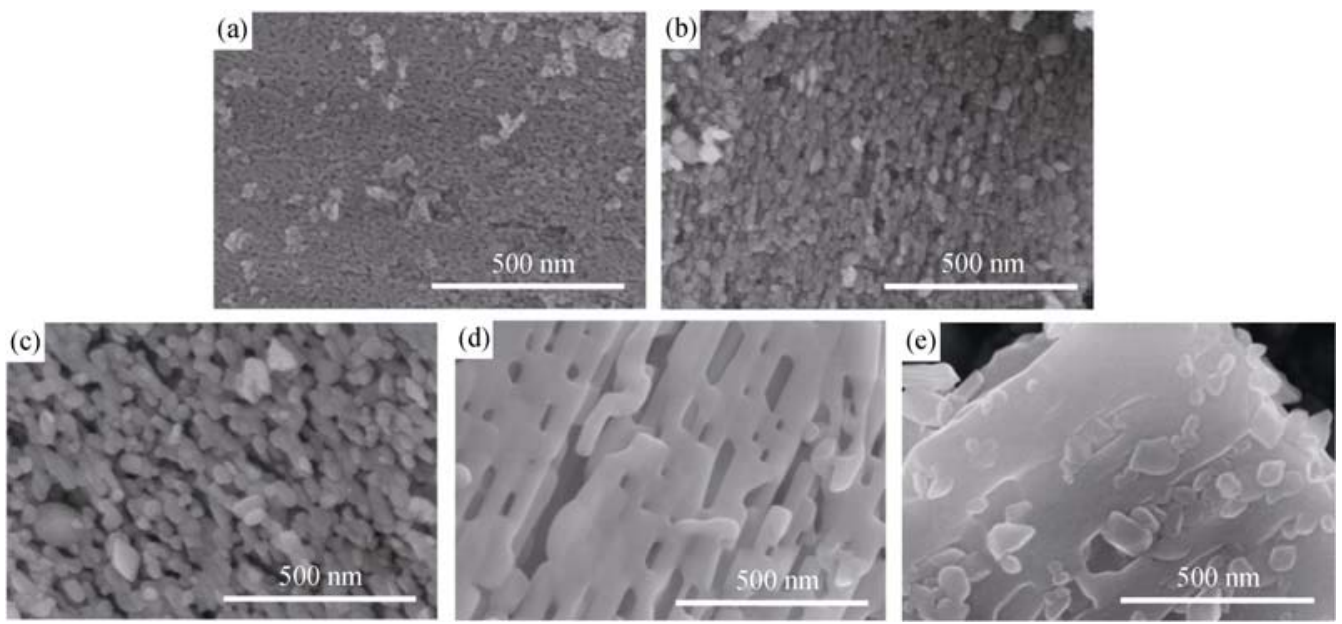

图 2 不同焙烧温度制备多孔钼钛氧化物的 FESEM 照片

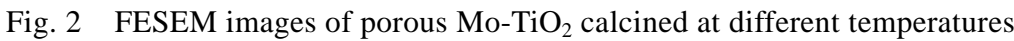
(a) $400{ }^{\circ} \mathrm{C}$; (b) $500^{\circ} \mathrm{C}$; (c) $600{ }^{\circ} \mathrm{C}$; (d) $700^{\circ} \mathrm{C}$; (e) $800^{\circ} \mathrm{C}$ 


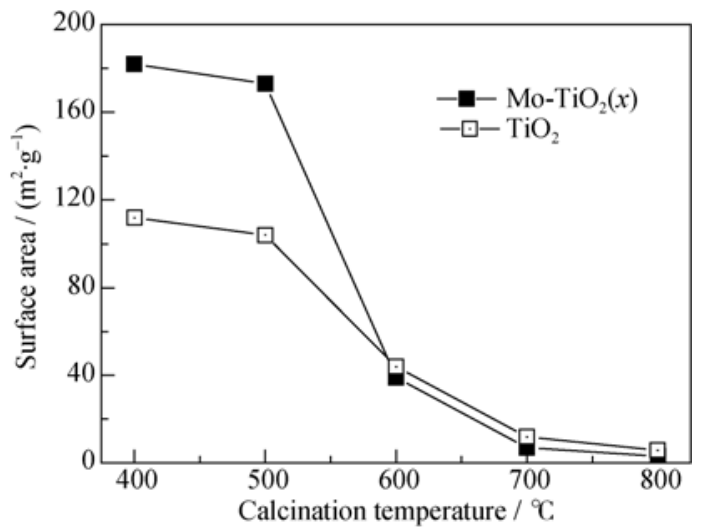

图 3 不同焙烧温度制备 $\mathrm{Mo}-\mathrm{TiO}_{2}$ 和 $\mathrm{TiO}_{2}$ 比表面积的比较

Fig. 3 Comparison on the surface area of $\mathrm{Mo}-\mathrm{TiO}_{2}$ and $\mathrm{TiO}_{2}$ calcined at different temperatures

所减小，自支撑 $\mathrm{Mo}-\mathrm{TiO}_{2}$ 的比表面积略低于相同温 度烧制的 $\mathrm{TiO}_{2}$, 表明当高于 $600^{\circ} \mathrm{C}$ 时，自支撑对氧 化物孔结构的维持效果减弱。

图 4 显示了不同多孔 $\mathrm{Mo}^{-} \mathrm{TiO}_{2}$ 的加氢脱硫性能 数据。如图所示, $\mathrm{Mo}^{-} \mathrm{TiO}_{2}(400)$ 表现出了最佳的加 氢脱硫性能, 其 DBT 转化率为 $80 \%, \mathrm{Mo}^{-} \mathrm{TiO}_{2}(500)$ 的 DBT 转化率达到 $62 \%, \mathrm{Mo}-\mathrm{TiO}_{2}(400)$ 和 $\mathrm{Mo}^{-} \mathrm{TiO}_{2}(500)$ 两组催化材料的加氢脱硫性能显著 优于没有自支撑制备的对比样品 (control sample), 其 DBT 转化率仅为 $48 \%$, 这主要与 $\mathrm{Mo}-\mathrm{TiO}_{2}(400)$ 和 $\mathrm{Mo}^{-} \mathrm{TiO}_{2}(500)$ 两组催化材料具有更高的比表面 积有关。高的比表面积不仅可以提供更多的反应界 面，而且还能负载更多的活性物质 ${ }^{[12,24]}$, 如表 1 所 示, 两者的 $\mathrm{MoO}_{3}$ 含量均高于对比样品。由此可知, 催化材料比表面积低, 活性组分负载量小, 进而导 致其加氢脱硫性能差, 故高温焙烧的 Mo- $\mathrm{TiO}_{2}(600) 、 \mathrm{Mo}-\mathrm{TiO}_{2}(700)$ 和 $\mathrm{Mo}-\mathrm{TiO}_{2}(800)$ 的 DBT 转化率低于对比样品。

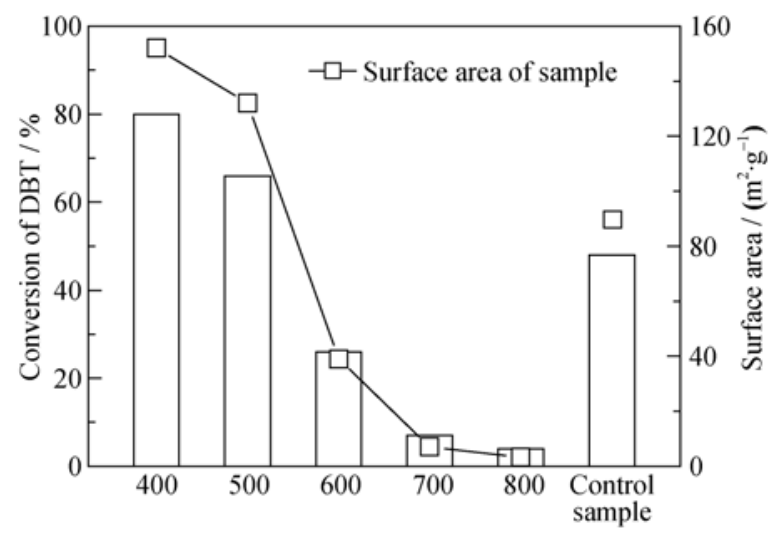

图 4 不同多孔钼钛氧化物和对比样品的加氢脱硫性能图 Fig. 4 Hydrodesulfurization performance of various porous $\mathrm{Mo}-\mathrm{TiO}_{2}$

\section{2 焙烧过程对孔结构的影响分析}

在多数情况下，材料的比表面积会随着其晶体 结构的转变而发生变化, 因此需要进一步分析各焙 烧温度制备的多孔钼钛氧化物的晶体结构。图 5 显 示 $\mathrm{Mo}^{-\mathrm{TiO}_{2}}(400)$ 和 $\mathrm{Mo}-\mathrm{TiO}_{2}(500)$ 在 $2 \theta=25.3^{\circ} 、 37.8^{\circ}$ 、 $48^{\circ}$ 处有三个微弱的衍射峰, 对应的是锐钛矿 $\mathrm{TiO}_{2}$ 的(101)、(004)和(200)晶面(JDSC: 21-1272), 这表明 两种钼钛氧化物中的 $\mathrm{TiO}_{2}$ 为锐钛矿相。在 Mo- $\mathrm{TiO}_{2}(600)$ 中的锐钛矿特征峰峰高增强, 但峰宽 减小, 说明其 $\mathrm{TiO}_{2}$ (见表 1) 较 $\mathrm{Mo}^{-} \mathrm{TiO}_{2}(400)$ 和 $\mathrm{Mo}^{-} \mathrm{TiO}_{2}(500)$ 发生晶粒生长, 从表 1 的晶粒尺寸数 据也可得到证明。此外, 在 $2 \theta=27.4^{\circ} 、 36.1^{\circ} 、 41.2^{\circ}$ 和 $54.3^{\circ}$ 处出现了四个金红石相的特征峰(JDSC: 21-1276), 表明在 $\mathrm{Mo}^{-} \mathrm{TiO}_{2}(600)$ 制备过程中发生了 锐钛矿向金红石的转变。根据李灿等报道 ${ }^{[17]}$, 锐钛 矿向金红石转变是由锐钛矿晶粒相互熔合形成了金 红石相, 这解释了在图 2 的 FESEM 中 $\mathrm{Mo}^{-} \mathrm{TiO}_{2}$ (600、700、800)样品出现晶粒熔合现象的原因。锐 钛矿向金红石的转晶会导致 $\mathrm{TiO}_{2}$ 比表面积急剧下 降 ${ }^{[25]}$, 因此, 这也解释了 $\mathrm{Mo}^{-} \mathrm{TiO}_{2}(600)$ 的比表面积 为何明显低于 $\mathrm{Mo}^{-} \mathrm{TiO}_{2}(400)$ 和 $\mathrm{Mo}^{-} \mathrm{TiO}_{2}(500)$ 。前期 研究结果已经证明, 由钛酸盐衍生物合成的 $\mathrm{TiO}_{2}$ 具 有相对较好的热稳定性，其锐钛矿向金红石相的转 变温度可高达 $800{ }^{\circ} \mathrm{C}^{[26]}$, 但是本实验结果显示 $\mathrm{Mo}-\mathrm{TiO}_{2}$ 的转变温度仅为 $600^{\circ} \mathrm{C}$, 当焙烧温度进一 步提升后，在 $\mathrm{Mo}-\mathrm{TiO}_{2}(700)$ 和 $\mathrm{Mo}-\mathrm{TiO}_{2}(800)$ 的 XRD 图谱中仅能观察到金红石型 $\mathrm{TiO}_{2}$ 。显然, 钼钛氧化 物中 $\mathrm{TiO}_{2}$ 转变温度的降低与 $\mathrm{MoO}_{3}$ 有关, 故需要研 究钿钛氧化物中 $\mathrm{MoO}_{3}$ 的状态。

如图 6 所示, 在 $2 \theta=12.8^{\circ} 、 23.3^{\circ} 、 25.7^{\circ} 、 27.3^{\circ}$ 、 $33.7^{\circ}$ 和 $39.1^{\circ}$ 处均出现强衍射峰, 分别对应的是正

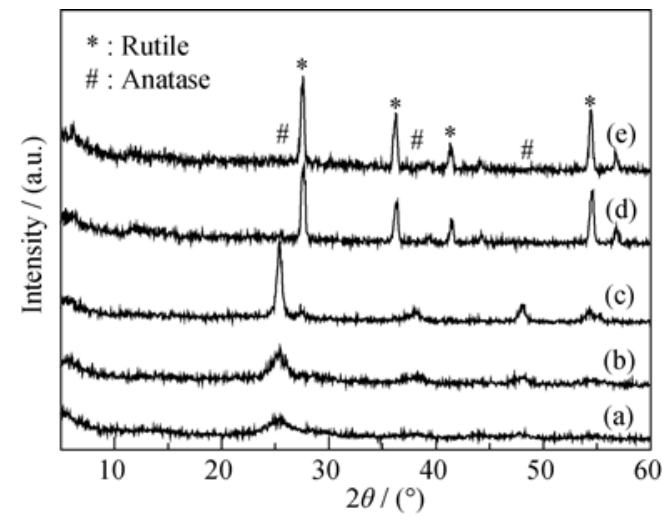

图 5 不同焙烧温度制备多孔钼钛氧化物的 XRD 图谱

Fig. 5 XRD patterns of porous $\mathrm{Mo}-\mathrm{TiO}_{2}$ calcined at different temperatures

(a) $400^{\circ} \mathrm{C}$; (b) $500^{\circ} \mathrm{C}$; (c) $600{ }^{\circ} \mathrm{C}$; (d) $700^{\circ} \mathrm{C}$; (e) $800^{\circ} \mathrm{C}$ 


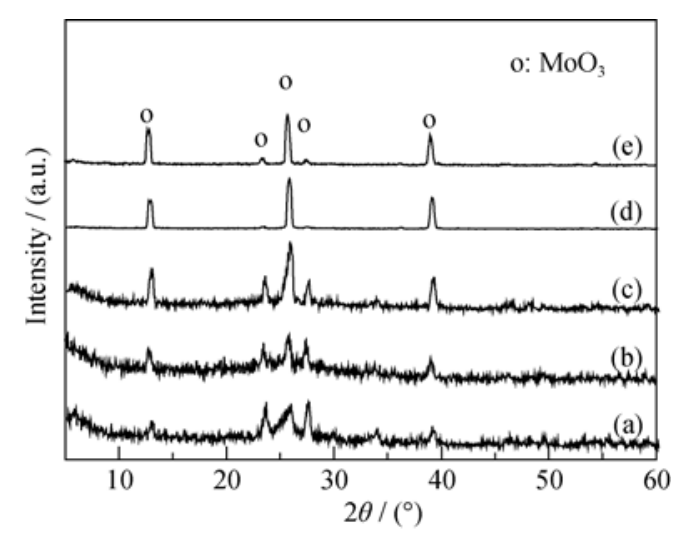

图 6 不同焙烧温度制备 $\mathrm{Mo}(\mathrm{I})-\mathrm{TiO}_{2}$ 前驱体的 XRD 图谱

Fig. 6 XRD patterns of precursor $\mathrm{Mo}(\mathrm{I})-\mathrm{TiO}_{2}$ calcined at different temperatures

(a) $400{ }^{\circ} \mathrm{C}$; (b) $500^{\circ} \mathrm{C}$; (c) $600{ }^{\circ} \mathrm{C}$; (d) $700^{\circ} \mathrm{C}$; (e) $800^{\circ} \mathrm{C}$

交相 $\mathrm{MoO}_{3}$ 的(200)、(101)、(400)、(210)、(111)和(600) 晶面 ${ }^{[27]}$, 未观察到锐钛矿的特征峰, 这归因于锐钛 矿在 $2 \theta=25^{\circ}$ 处信号最强的特征峰被 $\mathrm{MoO}_{3}$ 的特征峰 覆盖。所有样品的峰高均随着焙烧温度的升高而增 强, 而在同一样品中不同峰峰高的增加速率不同, 差别最大的是(101)、(400)和(210)这几个晶面所对应 的特征峰, (400)晶面的峰高(记作 $I(400)$ ) 随着焙烧温 度的升高而增加明显, 相比之下, 与(101)和(210)晶 面(记作 $I(101)$ 和 $I(210)$ ) 对应的峰高则增加较为缓慢, 表明 $\mathrm{MoO}_{3}$ 主要向(400)晶面方向生长, 这是正交相 $\mathrm{MoO}_{3}$ 晶体生长的典型特征 ${ }^{[28]}$ 。

进一步解析 $\mathrm{MoO}_{3}$ 生长与焙烧温度的关系, 可 以发现其 $I(400)$ 分别与 $I(101) 、 I(210)$ 的比值和焙烧 温度间存在一定的线性关系如图 7 所示。根据 JDSC: 47-1320, $\mathrm{MoO}_{3}$ 的 $I(400) / I(101)$ 和 $I(400) / I(210)$ 标准 比值分别为 1.11 和 1.22 , 标准比值与两条拟合曲线 间的交点大约为 $450^{\circ} \mathrm{C}$ 。众所周知, $\mathrm{MoO}_{3}$ 在 $450^{\circ} \mathrm{C}$ 时开始熔融, 其在 $\mathrm{TiO}_{2}$ 表面会以单分子层形式

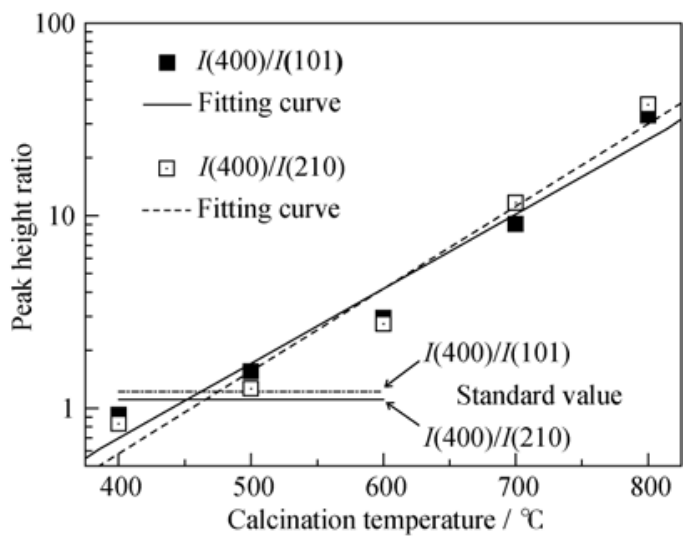

图 7 多孔钼钛氧化物的峰高比值对比图

Fig. 7 Peak height ratio of porous $\mathrm{Mo}^{-\mathrm{TiO}_{2}}$ composites
自发铺展 ${ }^{[29]}$ 。钼钛氧化物中 $\mathrm{MoO}_{3}$ 的晶体生长状态 可以通过峰高比值和标准值的比较来判断：当钼钛 氧化物的峰高比值低于标准值时, 表明 $\mathrm{MoO}_{3}$ 未发 生晶体生长, 意味着 $\mathrm{MoO}_{3}$ 在该条件下没有熔融; 若样品的峰高比值高于标准值, 表明在焙烧过程中 $\mathrm{MoO}_{3}$ 处于熔融状态。由此可知, 钿钛氧化物中的 $\mathrm{MoO}_{3}$ 在 $400^{\circ} \mathrm{C}$ 焙烧条件下呈固体状态, $\mathrm{Mo}-\mathrm{TiO}_{2}(500)$ 中 $\mathrm{MoO}_{3}$ 的峰高比值与标准比值接近, 表明 Mo- $\mathrm{TiO}_{2}$ (500)中的 $\mathrm{MoO}_{3}$ 在 $500^{\circ} \mathrm{C}$ 下大部分呈固体 状态, 且伴有少部分熔融。在 $\mathrm{Mo}^{-} \mathrm{TiO}_{2}(600)$ 、 $\mathrm{Mo}^{-\mathrm{TiO}_{2}}(700)$ 和 $\mathrm{Mo}-\mathrm{TiO}_{2}(800)$ 中 $\mathrm{MoO}_{3}$ 比值明显高 于标准值, 所以这几组样品的 $\mathrm{MoO}_{3}$ 在焙烧过程中 处于完全熔融状态。

通过热分析技术对钼钛氧化物的焙烧过程进行 研究, 钼酸铵与钛酸盐衍生物混合物的 TG 和 DSC 分析如图 8。TG 曲线显示钼酸铵与钛酸盐衍生物混 合物的失重主要分为两个阶段：物理吸附水的蒸 发、钼酸铵和钛酸盐衍生物结合水的脱除等过程均 发生在 $400^{\circ} \mathrm{C}$ 以下 ${ }^{[30]}$; 第二阶段始于 $700^{\circ} \mathrm{C}$ 而终于 $920^{\circ} \mathrm{C}$, 这个阶段失重非常明显, 主要归因于 $\mathrm{MoO}_{3}$ 的挥发 ${ }^{[31]}$ 。此外, TG 曲线显示混合物在 $400 \sim 700^{\circ} \mathrm{C}$ 间没有质量损失，但并不意味其在焙烧过程中没有 发生化学反应，从 DSC 曲线中可观察到混合物在该 温度区间内存在轻微的吸热过程, 主要归因于 $\mathrm{MoO}_{3}$ 的熔融吸热。熔融的 $\mathrm{MoO}_{3}$ 一方面无法为 $\mathrm{TiO}_{2}$ 孔道提供支撑, 另一方面可能为 $\mathrm{TiO}_{2}$ 的转晶提供了 液相环境, 这与某些陶瓷材料在烧制过程中熔盐的 作用相似 ${ }^{[32-33]}$ 。

\section{3 结构转变机理的探讨}

综合上述分析结果可知, 多孔钼钛氧化物的结 构主要受焙烧温度的影响。如图 1 和图 3 所示, 催 化材料的比表面积随着焙烧温度的升高而减少,

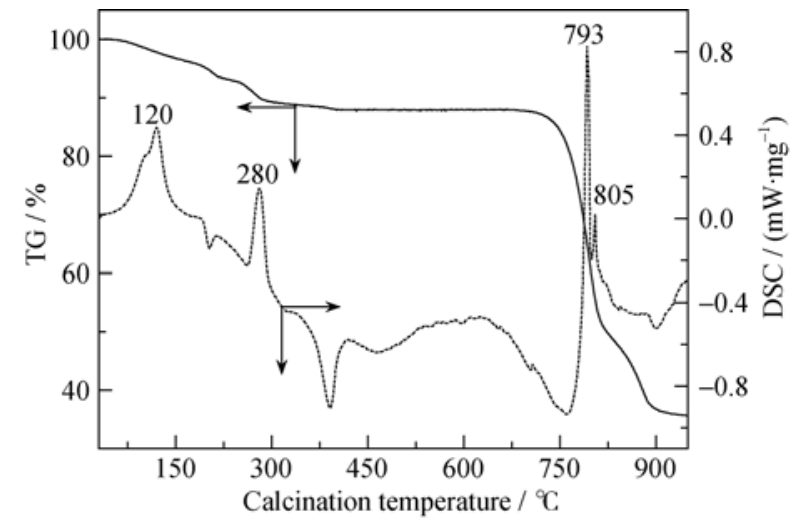

图 8 钼酸铵与钛酸盐衍生物混合物的 TG 和 DSC 曲线图 Fig. 8 TG and DSC curves of hybrid of ammonium molybdate and titanate derivative 


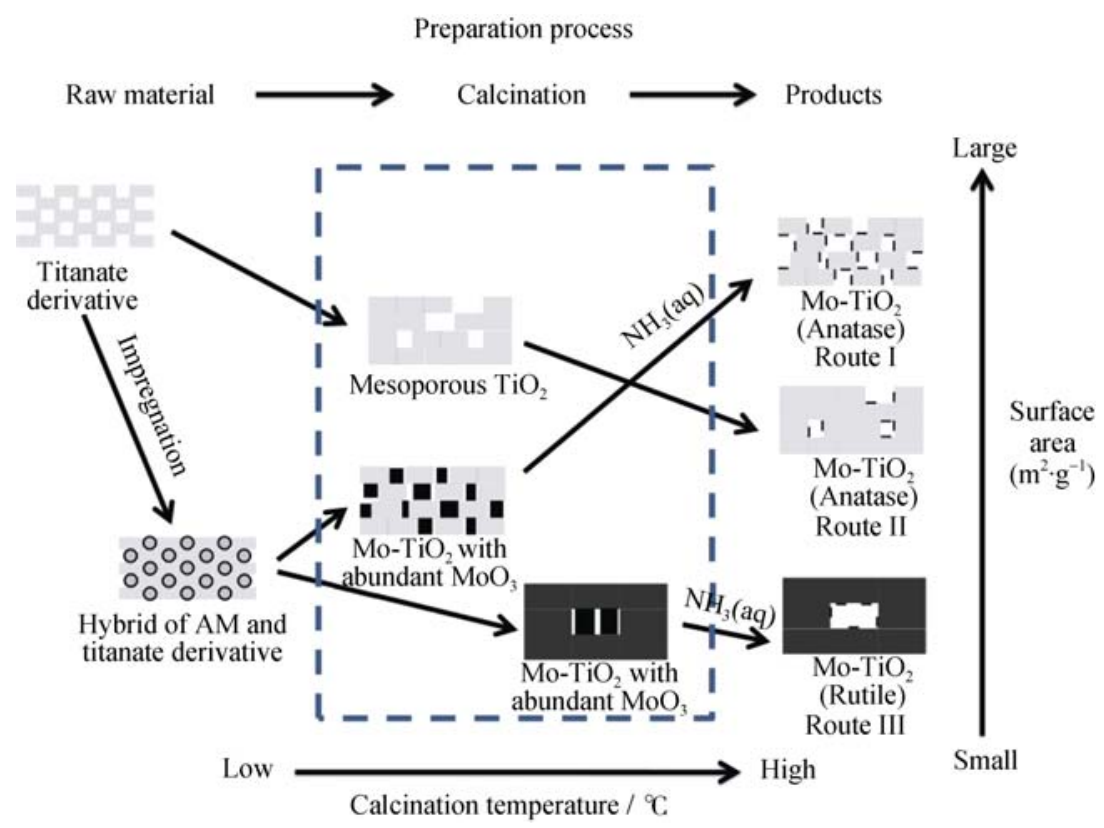

图 9 各种多孔钼钛氧化物的制备过程示意图

Fig. 9 Scheme for preparation process of various porous $\mathrm{Mo}-\mathrm{TiO}_{2}$ composites

当焙烧温度在 $500 \sim 600^{\circ} \mathrm{C}$ 之间时, 材料的比表面积 下降尤为明显, $\mathrm{XRD}$ 分析证明这主要是 $\mathrm{Mo}-\mathrm{TiO}_{2}$ 中 的 $\mathrm{TiO}_{2}$ 发生锐铁矿向金红石转晶所致, 而 $\mathrm{TiO}_{2}$ 的转 晶可能与 $\mathrm{MoO}_{3}$ 发生熔融有关。因此, 在焙烧温度 高于 $600^{\circ} \mathrm{C}$ 条件下所制得的钿钛氧化物(图 9, 制备 途径 III)比表面积低于没有自支撑作用得到的钿钛 氧化物(图 9, 制备途径 II)。与之相反, 焙烧温度为 $400^{\circ} \mathrm{C}$ 或 $500^{\circ} \mathrm{C}$ 时, $\mathrm{MoO}_{3}$ 主要呈固体状态, 大量的 $\mathrm{MoO}_{3}$ 在焙烧过程中可以保护 $\mathrm{TiO}_{2}$ 的孔结构, 用氨 水溶解掉前驱体中过量的 $\mathrm{MoO}_{3}$ 后, 可得到具有高 比表面积的钼钛氧化物(图 9, 制备途径 I)。

\section{3 结论}

本研究通过自支撑合成技术制备了一系列比 表面积从 3.2 $182 \mathrm{~m}^{2} / \mathrm{g}$ 的多孔钼钛氧化物, 高比表 面积的钼钛氧化物可以负载更多分散良好的 $\mathrm{MoO}_{3}$, 进而表现出更好的加氢脱硫性能。XRD 和 TG/DSC 分析发现钼钛氧化物的结构与 $\mathrm{MoO}_{3}$ 的状态有关, 在焙烧温度低于 $600{ }^{\circ} \mathrm{C}$ 时, 固体 $\mathrm{MoO}_{3}$ 可以支撑 $\mathrm{TiO}_{2}$ 孔道以防止其倜塌, 故制得的钼钛氧化物其 表面积显著高于没有自支撑的样品; 而当焙烧温 度升至 $600^{\circ} \mathrm{C}$ 及以上时, 钿钛氧化物中的 $\mathrm{MoO}_{3}$ 发 生熔化、挥发, 无法支撑 $\mathrm{TiO}_{2}$ 的孔道, 同时还促进 锐钛矿向金红石转变, 进而导致钼钛氧化物的孔 结构坍塌。

\section{参考文献:}

[1] RAMIREZ J, LUIS C, GUIDO B. The role of titania support in Mo-based hydrodesulfurization catalysts. Journal of Catalysis, 1999, 184(1): 59-67.

[2] CHEN X B, SAMUEL S M. Titanium dioxide nanomaterials: synthesis, properties, modifications, and applications. Chemical Reviews, 2007, 107(7): 2891-2959.

[3] DAHL M, LIU Y D, YIN Y D. Composite titanium dioxide nanomaterials. Chemical Reviews, 2014, 114(19): 9853-9889.

[4] ZHANG H Z, BANFIELD J F. Understanding polymorphic phase transformation behavior during growth of nanocrystalline aggregates: insights from $\mathrm{TiO}_{2}$. The Journal of Physical Chemistry B, 2000, 104(15): 3481-3487.

[5] HANAOR D A H, SORRELL C C. Review of the anatase to rutile phase transformation. Journal of Materials Science, 2011, 46(4): 855-874.

[6] MOHAMMADI M R, FRAY D J, MOHAMMADI A. Sol-Gel nanostructured titanium dioxide: controlling the crystal structure, crystallite size, phase transformation, packing and ordering. Microporous and Mesoporous Materials, 2008, 112(1): 392-402.

[7] ANTONELLI D M, JACKIE Y Y. Synthesis of hexagonally packed mesoporous $\mathrm{TiO}_{2}$ by a modified Sol-Gel method. Angewandte Chemie International Edition in English, 1995, 34(18): 2014-2017.

[8] LEE D W, LEE K H. Novel eco-friendly synthesis of sucrose-templated mesoporous titania with high thermal stability. 
Microporous and Mesoporous Materials, 2011, 142(1): 98-103.

[9] JUNG J H, HIDEKI K, KJELD J C B, et al. Creation of novel helical ribbon and double-layered nanotube $\mathrm{TiO}_{2}$ structures using an organogel template. Chemistry of Materials, 2002, 14(4): 1445-1447.

[10] KASUGA T, MASAYOSHI H, AKIHIKO H, et al. Formation of titanium oxide nanotube. Langmuir, 1998, 14(12): 3160-3163.

[11] CORTES J M A, ESCOBAR J, ANGELES C, et al. Highly dispersed CoMoS phase on titania nanotubes as efficient HDS catalysts. Catalysis Today, 2008, 130(1): 56-62.

[12] DZWIGAJ S, LOUIS C, BREYSSE M, et al. New generation of titanium dioxide support for hydrodesulfurization. Applied Catalysis B: Environmental, 2003, 41(1): 181-191.

[13] INOUE S, AKIHIRO M, HIDEHIKO K, et al. Preparation of novel titania support by applying the multi-gelation method for ultradeep HDS of diesel oil. Applied Catalysis A: General, 2004, 269(1): 7-12.

[14] HE M, LU X H, FENG X, et al. A simple approach to mesoporous fibrous titania from potassium dititanate. Chemical Communications, 2004(19): 2202-2203.

[15] OKADA K, NOBUO Y, YOSHIKAZU K, et al. Effect of silica additive on the anatase-to-rutile phase transition. Journal of the American Ceramic Society, 2001, 84(7): 1591-1596.

[16] YANG J, HUANG Y X, Ferreira J M F. Inhibitory effect of alumina additive on the titania phase transformation of a Sol--Gel-derived powder. Journal of Materials Science Letters, 1997, 16(23): 1933-1935.

[17] ZHANG J, Li M J, FENG Z C, et al. UV Raman spectroscopic study on $\mathrm{TiO}_{2}$. I. phase transformation at the surface and in the bulk. The Journal of Physical Chemistry B, 2006, 110(2): 927-935.

[18] YUAN C Z, ZHOU L, HOU L R. Facile fabrication of self-supported three-dimensional porous reduced graphene oxide film for electrochemical capacitors. Materials Letters, 2014, 124: 253-255.

[19] WANG Z, Chen G, DING K. Self-supported catalysts. Chemical Reviews, 2008, 109(2): 322-359.

[20] MAKWANA N M, RAUL Q C, PARKIN I P, et al. A simple and low-cost method for the preparation of self-supported $\mathrm{TiO}_{2}-\mathrm{WO}_{3}$ ceramic heterojunction wafers. Journal of Materials Chemistry A, 2014, 2(41): 17602-17608.

[21] SUN J B, LI B, CAI K P, et al. $\mathrm{TiO}_{2}$ Photolysis device fabricated by direct ink write assembly. Journal of Inorganic Materials, 2011, 26(3): 300-304.

[22] SPURR R A, HOWARD M. Quantitative analysis of anatase-rutile mixtures with an X-ray diffractometer. Analytical Chemistry, 1957, 29(5): 760-762.

[23] SHENG Q R, YUAN S, ZHANG J L, et al. Synthesis of mesoporous titania with high photocatalytic activity by nanocrystalline particle assembly. Microporous and Mesoporous Materials, 2006, 87(3): 177-184.

[24] LI L C, WANG Y F, SHI K Z, et al. Preparation and characterization of mesoporous $\mathrm{MoO}_{3} / \mathrm{TiO}_{2}$ composite with high surface area by self-Supporting and ammonia method. Catalysis Letters, 2012, 142: 480-485.

[25] ZHANG J, XU Q, FENG Z C, et al. Importance of the relationship between surface phases and photocatalytic activity of $\mathrm{TiO}_{2}$. Angewandte Chemie International Edition in English, 2008, 47(9): 1766-1769.

[26] LI W, BAI Y, LIU C, et al. Highly thermal stable and highly crystalline anatase $\mathrm{TiO}_{2}$ for photocatalysis. Environmental Science \& Technology, 2009, 43(14): 5423-5428.

[27] SONG R Q, XU A W, DENG B, et al. Novel multilamellar mesostructured molybdenum oxide nanofibers and nanobelts: synthesis and characterization. The Journal of Physical Chemistry B, 2005, 109(48): 22758-22766.

[28] BADICA P. Preparation through the vapor transport and growth mechanism of the first-order hierarchical structures of $\mathrm{MoO}_{3}$ belts on sillimanite fibers. Crystal growth \& design, 2007, 7(4): 794-801.

[29] ONO T, KAMISUKI H, HISASHI H, et al. A comparison of oxidation activities and structures of Mo oxides highly dispersed on group IV oxide supports. Journal of Catalysis, 1989, 116(1): 303-307.

[30] THORNE A, KRUTH A, TUNSTALL D, et al. Formation, structure, and stability of titanate nanotubes and their proton conductivity. The Journal of Physical Chemistry B, 2005, 109(12): 5439-5444.

[31] SHAHEEN W M, SELIM M M. Thermal decompositions of pure and mixed manganese carbonate and ammonium molybdate tetrahydrate. Journal of Thermal Analysis and Calorimetry, 2000, 59(3): 961-970.

[32] ZENG T, BAI Y, LI H, et al. Preparation of polyhedral copper oxide nanoparticles by molten-salt method and their catalytic performance. Journal of Inorganic Materials, 2015, 30(4): 439-442.

[33] WU X, LI H F, ZHOU J L, et al. Fabrication and properties of highly pure $\mathrm{BiFeO}_{3}$ using a method of solid state reaction-molten salt synthesis with non-equilibrium Process. Journal of Inorganic Materials, 2014, 29(11): 1151-1155. 